\title{
Understanding how statins work: the path to better treatments for heart disease and more
}
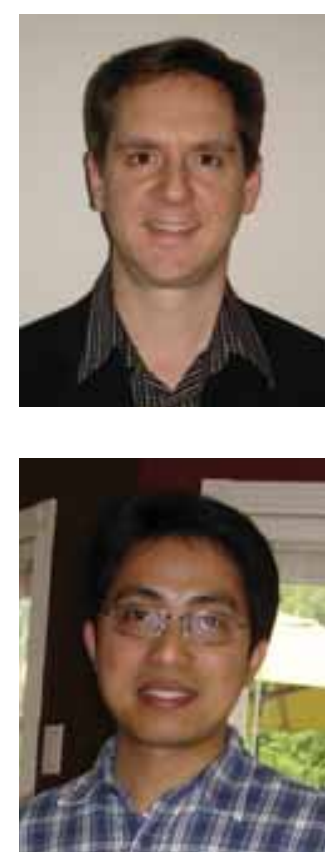

\section{Jom SHillt \& GuosongQiu}

${ }^{\dagger}$ Author for correspondence Atherosclerosis Specialty Laboratory, $\mathrm{H}$ ealthy $\mathrm{H}$ eart Program, St Paul's H ospital, James H ogg iCAPT U RE Centre for Cardiovascular and Pulmonary Research, $D$ epartment of Pathology and Laboratory M edicine, University of British Columbia, Vancouver, BC, Canada

Tel.: +1 6048068616 ; Fax: +1 6048068590 ; jshill@interchange.ubc.ca '...sta tin trea tment a c hieves 80-100\% success for a $30 \%$ reduction in LDL-cholesterol in primary prevention...'

The increasing use of 3-hydroxy-3-methylglutaryl (H M G)-C OA reductase inhibitors (statins) has revealed beneficial effects that are independent of their intended lipid-lowering effects. Understanding the mechanisms of how statins facilitate these effects may help extend their indications in both cardiovascular and noncardiovascular diseases and perhaps lead to the development of additional novel pharmaceuticals.

$O$ wing to their structural similarity with HMG-COA, statins are able to competitively inhibit HMG-CoA reductase, the rate-limiting enzyme in cholesterol synthesis. The consequence of this inhibition is a reduction in cholesterol synthesis and subsequent secretion of ApoB-containing lipoproteins and an increase in the clearance of LDL-cholesterol through the upregulation of the LDL receptor. A variety of clinical studies have demonstrated the efficacy of statins in LDL-cholesterol lowering, a reduction that is approximately two-to-four-times as effective as observed with fibrate treatment. In general, statin treatment achieves $80-100 \%$ success for a $30 \%$ reduction in LDL-cholesterol in primary prevention, which corresponds to an approximately $30 \%$ reduction in cardiovascular events, including acute coronary syndrome (ACS), myocardial infarction and stroke for secondary prevention [1-5].

Nonlipid-lowering effects of statins

Several clinical trials including $\mathrm{H}$ eart Protection Study (H PS), Collaborative Atorvastatin Diabetes Study (CARD S) and Treating to $\mathrm{N}$ ew Targets (TNT) Study have suggested that statins can offer protection to patients with normal lipid levels through various nonlipid-lowering effects. For example, statins can upregulate or recover nitric oxide synthase expression in endothelia, not only in the absence of risk factors, but also when treated with oxidized LDL (OxLDL), TNF- $\alpha$ and during hypoxic conditions. Clinically, statins have been shown to restore endothelial function, improve flow-mediated dilation and blood flow of the brachial artery within $24 \mathrm{~h}$, independent of changes in LDL [6].

Statins also have inherent anti-inflammatory abilities, as treatment can reduce the expression of adhesion molecules and chemokines such as ICAM -1, integrin adhesion molecules, M CP-1, IL-8, CX3CL1 and CX3CR1, and thereby decrease subsequent inflammatory cell adhesion to endothelia and production of proinflammatory cytokines [7]. C-reactive protein (CRP), a well-recognized inflammatory biomarker, is also reduced by statins in human hepatocytes, leading to a lower CRP level as reported in the Pravastatin or Atorvastatin Evaluation and Infection Therapy (PROVE IT-TIMI 22) trial [8-10].

'High-dose statin therapy has been demonstrated to stop or even regress plaque progression...'

O xidative stress can also be ameliorated with statin therapy. Statins negatively regulate the formation of the $\mathrm{NAD}(\mathrm{P}) \mathrm{H}$ oxidase subunit p22phox- and myeloperoxidase-derived reactive oxygen species [11,12]. Furthermore, statins strengthen systemic antioxidant effects through the upregulation of heme oxygenase-1, catalase, superoxide dismutase, glutathione peroxidase and paraoxonase 1 expression in a variety of cells, promoting protection against oxidative stress [13]. Coincident with decreased plasma endogenous peroxide concentrations and increased total antioxidant capacity, antibody titers to malondialdehyde-LDL, Cu-OxLDL and OxLDL are also decreased in patients receiving statin therapy [14].

Statins have also been reported to stabilize atherosclerotic plaques and may, in fact, cause their regression. M atrix metalloproteinase production, which has been related to the thinning of the fibrous cap and plaque rupture, can be effectively suppressed by statins in macrophages and smooth muscle cells [15]. In addition, statin therapy represses plaque neovascularization and the COX-2/microsomal 
prostaglandin E synthase-1 proinflammatory pathway, which favor plaque destabilization. Statins are also well-known, potent inhibitors of smooth muscle cell migration and proliferation, and sensitize human smooth muscle cells to undergo apoptosis. High-dose statin therapy has been demonstrated to stop or even regress plaque progression as reported in the Reversing Atherosclerosis with Aggressive Lipid Lowering (REVERSAL), M easuring Effects on IntimaMedia Thickness: an Evaluation of Rosuvastatin (METEOR) and A Study to Evaluate the Effect of Rosuvastatin on Intravascular U Itrasound-Derived Coronary Atheroma Burden (AST EROID) studies [16-18].

$M$ any studies have provided evidence that statins can effectively interfere with thrombosis through:

- Inactivation of coagulation

- Platelet inactivation

- Fibrinolytic activation

In coronary artery disease patients, statins slow blood clotting corresponding to decreased thrombin formation and attenuated coagulation factor activation [19]. Furthermore, statins inhibit the activity and antigen level of the platelet proteinase-activated receptor-1 thrombin receptor, CD 40L, von Willebrand factor and tissue factors, resulting in reduced platelet aggregability. Finally, statin treatment drives the fibrinolytic process by upregulating tissue plasminogen activator and downregulating plasminogen activator inhibitor-1 [20], so as to remove fibrin from injured endothelial surfaces and eliminating the nidus for platelet aggregation and the cascade effect of the thrombotic response.

The immunomodulatory properties of statins is the focus of recent research. Statins particularly affect monocyte functions by blunting the expression of Toll-like receptor 4 and 2 [21,22]. The activation of $T$ lymphocytes, natural killer cells and dendritic cells, and the subsequent production of lymphokines are also attenuated by statin treatment. In addition, statins promote $T$ helper cell (Th)2 polarization through both the inhibition of the IFN- $\gamma$-producing Th1 response and augmentation of the Th2 development of $C D 4^{+} T$ cells [23]. Furthermore, the expression of M HC-II molecules in macrophages and endothelial cells, which are directly involved in antigen presenting and T-lymphocyte activation, is also effectively suppressed by statins [24]. In addition, reduced cell-surface expression of other immunoregulatory molecules, including M H C -I, CD 3, -4, -8, -28, -40, $-80,-86$ and -54 by statins influences lymphocyte homing and the subsequent immune response [25].

How do statins exert

nonlipid-lowering effects?

Statin treatment reduces the production of intermediates for cholesterol synthesis, especially isoprenoids such as farnesyl pyrophosphate and geranylgeranylpyrophosphate. These intermediates can influence many intracellular signaling pathways through the prenylation of small $G$ proteins. Thus far, small $G$ proteins have been found to be involved in the regulation of MAPKs (ERK, c-Jun $\mathrm{N}$-terminal kinase, P38 MAPK), PI3K-Akt and protein kinase $\mathrm{C}$-nuclear factor- $\kappa \mathrm{B}$ pathways.

Two important intranuclear receptor pathways for the pathognesis of cardiovascular diseases, namely liver $X$ receptors (LXRs) and PPARs are additional targets of statin treatment. Cholesterol derivatives such as oxysterols are recognized ligands for LXRs. The reduction of oxysterols consequent to statin therapy is accompanied by the corresponding inactivation of LXRs, causing altered expression of downstream genes such as lipases [26]. Statin treatment is also able to activate PPAR- $\alpha$ and $-\gamma$. The mechanism by which statins activate PPARs, however, remains unclear.

\footnotetext{
'Statin administration is a lso a ssocia ted with improved cardiac function and reduc ed a mhythmia episodes in both ischemic and nonischemic diseases.'
}

New applications of statins

Recently, new applications of statins in cardiovascular disease, such as ischemia-reperfusion injury, heart failure (HF) and arrhythmia have been documented in several clinical trials. In Aggrastat to Zocor (A to Z), Effects of Atorvastatin on Early Recurrent Ischemic Events in Acute Coronary Syndromes (MIRACL) and PROVE IT-TIMI 22 trials, the early initiation of an aggressive statin regimen within 24-96 h among patients with ACS resulted in reduced myocardial ischemia-reperfusion injury and a favorable reduction in major cardiovascular events. However, delayed initiation of statin therapy, even within 14 days after ACS, did not confer benefits up to 4 months. 
Statin administration is also associated with improved cardiac function and reduced arrhythmia episodes in both ischemic and nonischemic diseases. This treatment reduces the rate of hospitalization for H F by $27 \%$ [27]. In patients with dilated cardiomyopathy or implantable cardioverter defibrillator implantation, statins reduce the risk of arrhythmias (particularly malignant ventricular arrhythmia). Furthermore, pre or concomitant treatment with statins significantly reduces the incidence of atrial fibrillation after acute elective cardiac surgery [28,29].

Statins may also deliver beneficial effects on restenosis prevention after percutaneous coronary intervention and aortic stenosis, for which large-scaled studies are underway [30,31]. Moreover, the immunomodulatory property of statins can potentially ameliorate cardiac allograft rejection, resulting in improved survival and a significantly lower incidence of transplant vasculopathy [32]. I mmunomodulation by statins may also influence the course of immunerelated diseases, such as Alzheimer's disease/dementia, multiple sclerosis, inflammatory bowel diseases, rheumatic arthritis and ankylosing spondylitis. However, at present, no definitive conclusions can be drawn until large-scale clinical trials have been completed.

\section{Future perspective}

There is abundant evidence to suggest that statins have a variety of influences beyond their initially intended lipid-lowering effects. There is great value in elucidating the specific mechanisms of these effects as it is this knowledge that should guide the application of this class of drugs in patients in different clinical settings. The lack of adverse side effects for this class of compounds also makes it an attractive adjunctive therapy in specific applications. The future appears bright for statin therapy as its applications and benefits continue to multiply.

Financial \& competing interests disclosure The authors have no relevant affiliations or financial involvement with any organization or entity with a financial interes in or financial conflict with the subject matter or materials discussed in the manuscript. This includes employment, consultancies, honoraria, stock ownership or options, expert testimony, grants or patents received or pending, or royalties.

$\mathrm{No}$ writing assistance was utilized in the production of this manuscript.

\section{Bibliography}

1. Pedersen $T R$, Kjekshus J, Berg $K$ et al.: Randomised trial of cholesterol lowering in 4444 patients with coronary heart disease: the Scandinavian Simvastatin Survival Study (4S). Atheroscler. Suppl. 344(8934), 1383-1389 (1994).

2. Prevention of cardiovascular events and death with pravastatin in patients with coronary heart disease and a broad range of initial cholesterol levels. The LongTerm Intervention with Pravastatin in Ischaemic D isease (LIPID) Study Group N . Engl. J. M ed. 339(19), 1349-1357 (1998).

3. D owns JR, Clearfield $M$, Weis $S$ et al.: Primary prevention of acute coronary events with lovastatin in men and women with average cholesterol levels: results of AFCAPS/TexCAPS. Air Force/Texas C oronary Atherosclerosis Prevention Study. JAM A 279(20), 1615-1622 (1998).

4. Shepherd J, Blauw GJ, M urphy M B et al.: Pravastatin in elderly individuals at risk of vascular disease (PRO SPER): a randomised controlled trial. Lancet 360(9346), 1623-1630 (2002).
5. Shepherd J, Cobbe SM, Ford I et al.: Prevention of coronary heart disease with pravastatin in men with hypercholesterolemia. West of Scotland Coronary Prevention Study Group. N . Engl. J. M ed. 333(20), 1301-1307 (1995).

6. Beckman JA, Liao JK, H urley S et al.: Atorvastatin restores endothelial function in normocholesterolemic smokers independent of changes in low-density lipoprotein. Circ. Res. 95(2), 217-223 (2004).

7. Forrester JS, Libby P: The inflammation hypothesis and its potential relevance to statin therapy. Am. J. Cardiol. 99(5), 732-738 (2007).

8. Arnaud C, Burger F, Steffens $S$ et al.: Statins reduce interleukin-6-induced $C$-reactive protein in human hepatocytes: new evidence for direct antiinflammatory effects of statins. Arterioscler. Thromb. Vasc. Biol. 25(6), 1231-1236 (2005).

9. Mayer $C$, Gruber HJ, Landl EM et al.: Rosuvastatin reduces interleukin-6-induced expression of $C$-reactive protein in human hepatocytes in a STAT 3- and C/EBPdependent fashion. Int. J. Clin. Pharmacol. Ther. 45(6), 319-327 (2007).
10. Ray KK, Cannon CP, Cairns R et al.: Relationship between uncontrolled risk factors and $C$-reactive protein levels in patients receiving standard or intensive statin therapy for acute coronary syndromes in the PRO VE IT-TIM I 22 trial. J. Am. Coll. Cardiol. 46(8), 1417-1424 (2005).

11. Rueckschloss U, Galle J, H oltz J et al.: Induction of N AD (P)H oxidase by oxidized low-density lipoprotein in human endothelial cells: antioxidative potential of hydroxymethylglutaryl coenzyme A reductase inhibitor therapy. Circulation 104(15), 1767-1772 (2001).

12. Kumar AP, Reynolds W F: Statins downregulate myeloperoxidase gene expression in macrophages. Biochem. Biophys. Res Commun. 331(2), 442-451 (2005).

13. D avignon J, Jacob RF, M ason RP: The antioxidant effects of statins. Coron. Artery. Dis. 15(5), 251-258 (2004)

14. Resch U, Tatzber F, Budinsky A et al.: Reduction of oxidative stress and modulation of autoantibodies against modified low-density lipoprotein after rosuvastatin therapy. Br. J. Clin. Pharmacol. 61(3), 262-274 (2006). 
15. Luan Z, C hase AJ, N ewby AC: Statins inhibit secretion of metalloproteinases-1, -2 , -3 , and -9 from vascular smooth muscle cells and macrophages. Arterioscler. Thromb. Vasc. Biol. 23(5), 769-775 (2003).

16. Nissen $S E$, Tuzcu EM , Schoenhagen $P$ et al.: Effect of intensive compared with moderate lipid-lowering therapy on progression of coronary atherosclerosis: a randomized controlled trial. JAM A 291(9), 1071-1080 (2004).

17. N issen SE, N icholls SJ, Sipahi I et al.: Effect of very high-intensity statin therapy on regression of coronary atherosclerosis: the ASTEROID trial. JAM A 295(13), 1556-1565 (2006).

18. CrouseJR 3rd, Raichlen JS, Riley WA et al.: Effect of rosuvastatin on progression of carotid intima-media thickness in low-risk individuals with subclinical atherosclerosis: the METEOR trial. JAM A 297(12), 1344-1353 (2007).

19. Undas A, Celinska-Lowenhoff M , Brummel-Ziedins KE et al.: Simvastatin given for 3 days can inhibit thrombin generation and activation of factor $V$ and enhance factor Va inactivation in hypercholesterolemic patients. Arterioscler. Thromb. Vasc. Biol. 25(7), 1524-1525 (2005).

20. M ussoni $L$, Banfi $C$, Sironi $L$ et al.: Fluvastatin inhibits basal and stimulated plasminogen activator inhibitor 1 , but induces tissue type plasminogen activator in cultured human endothelial cells. Thromb. $H$ aemost. 84(1), 59-64 (2000)

21. N iessner A, Steiner S, Speidl W S et al.: Simvastatin suppresses endotoxin-induced upregulation of Toll-like receptors 4 and 2 in vivo. Atherosclerosis 189(2), 408-413 (2006).

22. M ethe H, Kim JO, Kofler $\mathrm{S}$ et al.: Statins decrease Toll-like receptor 4 expression and downstream signaling in human $\mathrm{CD} 14^{+}$monocytes. Arterioscler. Thromb. Vasc. Biol. 25(7), 1439-1445 (2005).

23. H akamada-Taguchi $R$, U ehara $Y$, Kuribayashi $K$ et al.: Inhibition of hydroxymethylglutaryl-coenzyme a reductase reduces $\mathrm{T}$ hl development and promotes Th2 development. Circ. Res. 93(10), 948-956 (2003).

24. Geissler I, Collins L, Schofield R et al.: In vivo suppression of major histocompatibility complex class II expression on porcine vascular endothelial cells by an H M G-C oA reductase inhibitor. Transplantation 81(6), 922-926 (2006).

25. Kuipers H F, Biesta PJ, Groothuis TA et al.: Statins affect cell-surface expression of major histocompatibility complex class II molecules by disrupting cholesterolcontaining microdomains. H um. Immunol. 66(6), 653-665 (2005).

26. Q iu G, H ill JS: Atorvastatin decreases lipoprotein lipase and endothelial lipase expression in human TH P-1 macrophages. J. Lipid. Res. 48(10), 2112-2122 (2007).

27. Scirica BM , M orrow DA, Cannon CP et al.: Intensive statin therapy and the risk of hospitalization for heart failure after an acute coronary syndrome in the PRO VE ITTIMI 22 study. J. Am. Coll. Cardiol. 47(11), 2326-2331 (2006).

28. Patti $G, C$ Chello $M, C$ andura $D$ et al.: Randomized trial of atorvastatin for reduction of postoperative atrial fibrillation in patients undergoing cardiac surgery: results of the ARM YDA-3 (Atorvastatin for Reduction of M Yocardial D ysrhythmia After cardiac surgery) study. Circulation 114(14), 1455-1461 (2006).

29. Ramani $G, Z$ ahid M , Good CB et al.: Comparison of frequency of new-onset atrial fibrillation or flutter in patients on statins versus not on statins presenting with suspected acute coronary syndrome. Am. J. Cardiol. 100(3), 404-405 (2007).

30. Chan KL, Teo K, Tam J et al.: Rationale, design, and baseline characteristics of a randomized trial to assess the effect of cholesterol lowering on the progression of aortic stenosis: the Aortic Stenosis Progression $O$ bservation: M easuring Effects of R osuvastatin (AST RO N O M ER) trial. Am. H eart. J. 153(6), 925-931 (2007).

31. Rossebo $A B$, Pedersen $T R$, Allen $C$ et al.: $D$ esign and baseline characteristics of the simvastatin and ezetimibe in aortic stenosis (SEAS) study. Am. J. Cardiol. 99(7), 970-973 (2007).

32. WenkeK, M eiser B, ThieryJ et al.: Simvastatin initiated early after heart transplantation: 8-year prospective experience. Circulation 107(1), 93-97 (2003).

Affiliations

- John SHill Atherosclerosis Specialty L aboratory, H ealthy H eart Program, St Paul's H ospital, James H ogg iCAPTURE Centre for Cardiovascular and Pulmonary Research, D epartment of Pathology and Laboratory M edicine, U niversity of British Columbia, Vancouver, BC, Canada Tel.: +1 6048068616 ; Fax: +1 6048068590 ; jshill@interchange.ubc.ca

- Guosong Qiu Atherosclerosis Specialty L aboratory, H ealthy H eart Program, St Paul's H ospital, James H ogg iCAPTURE Centre for Cardiovascular and Pulmonary Research, D epartment of Pathology and Laboratory M edicine, U niversity of British Columbia, Vancouver, BC, Canada Tel.: +1 6048068616 ; Fax: +1 6048068590 ; gsqiu@panagin.com 